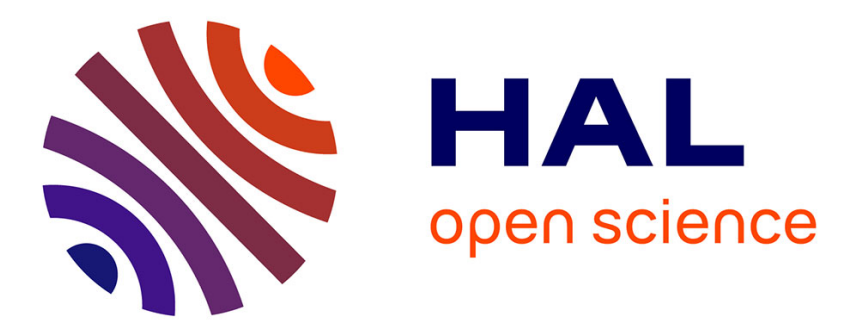

\title{
Persistence of incomplete mixing: A key to anomalous transport
}

\author{
Tanguy Le Borgne, Marco Dentz, Philippe Davy, Diogo Bolster, Jesus \\ Carrera, Jean-Raynald de Dreuzy, Olivier Bour
}

\section{To cite this version:}

Tanguy Le Borgne, Marco Dentz, Philippe Davy, Diogo Bolster, Jesus Carrera, et al.. Persistence of incomplete mixing: A key to anomalous transport. Physical Review E : Statistical, Nonlinear, and Soft Matter Physics, 2011, 84 (1, part 2), pp.n015301. 10.1103/PhysRevE.84.015301 . insu-00620762

\section{HAL Id: insu-00620762 \\ https://hal-insu.archives-ouvertes.fr/insu-00620762}

Submitted on 9 Mar 2021

HAL is a multi-disciplinary open access archive for the deposit and dissemination of scientific research documents, whether they are published or not. The documents may come from teaching and research institutions in France or abroad, or from public or private research centers.
L'archive ouverte pluridisciplinaire HAL, est destinée au dépôt et à la diffusion de documents scientifiques de niveau recherche, publiés ou non, émanant des établissements d'enseignement et de recherche français ou étrangers, des laboratoires publics ou privés. 


\title{
Persistence of incomplete mixing: A key to anomalous transport
}

\author{
Tanguy Le Borgne, ${ }^{1}$ Marco Dentz, ${ }^{2}$ Philippe Davy, ${ }^{1}$ Diogo Bolster, ${ }^{3}$ Jesus Carrera, ${ }^{2}$ \\ Jean-Raynald de Dreuzy, ${ }^{1}$ and Olivier Bour ${ }^{1}$ \\ ${ }^{1}$ Laboratoire Géosciences Rennes, Unité Mixte de Recherche CNRS Université de Rennes 1, F-35042 Rennes Cedex, France \\ ${ }^{2}$ Institute of Environmental Assessment and Water Research, Consejo Superior de Investigaciones Científicas, Barcelona, Spain \\ ${ }^{3}$ Environmental Fluid Dynamics Laboratory, University of Notre Dame, South Bend, Indiana, 46556, USA
}

(Received 6 August 2010; revised manuscript received 6 February 2011; published 20 July 2011)

\begin{abstract}
Anomalous dispersion in heterogeneous environments describes the anomalous growth of the macroscopic characteristic sizes of scalar fields. Here we show that this phenomenon is closely related to the persistence of local scale incomplete mixing. We introduce the mixing scale $\epsilon$ as the length for which the scalar distribution is locally uniform. We quantify its temporal evolution due to the competition of shear action and diffusion and compare it to the evolution of the global dispersion scale $\sigma$. In highly heterogeneous flow fields, for which the temporal evolution of $\sigma$ is superdiffusive, we find that $\epsilon$ evolves subdiffusively. The anomalous evolutions of the dispersion and mixing scales are complementary, $\epsilon \sigma \propto t$. This result relates anomalous global dispersion to the dynamics of local mixing.
\end{abstract}

DOI: 10.1103/PhysRevE.84.015301

PACS number(s): 47.51.+a, 05.60.Cd, 47.53.+n

Anomalous dispersion has been ubiquitously observed for transport in complex environments [1]. It can be traced back to the fact that projection of microscale transport dynamics in terms of stochastic or spatial averaging leads to non-Markovian behavior [2,3]. For Markovian systems, the memory of previous states is destroyed by complete mixing on the support scale. Mean field approaches typically rely on support scales that are not well mixed or, in other words, that are not in local equilibrium $[2,4]$. These approaches are characterized by nonMarkovian dynamics that (i) can lead to anomalous dispersion and (ii) may not preserve the full concentration variability. The latter is of critical importance for the quantification of phenomena that depend nonlinearly on local concentration such as reactive transport [4,5] or population biology [6,7]. Thus the understanding of anomalous dispersion and the quantification of concentration variability in heterogeneous environments depend on the notion of incomplete mixing and the evolution of the local mixing scale compared to the support scale of the projected transport dynamics. For example, as the mixing scale reaches the support scale, the projected transport is expected to become Markovian and represent the full spectrum of concentration values.

For homogeneous environments, mixing is due to diffusion only and the local mixing scale grows like $\sqrt{t}$. For heterogeneous environments, the mixing dynamics are more complex and are governed by the creation of concentration gradients due to heterogeneity and its destruction by microscale mixing. The dynamics of mixing have been studied for turbulent flows [8-10], chaotic flows [11,12], and heterogeneous porous media flows [13] in terms of global mixing states, as quantified by scalar dissipation rates [8], entropy measures [14], Lyapunov exponents [13], and pair dispersion [15]. While these approaches give invaluable insight into the mechanisms of mixing, they do not provide a quantification of a local mixing scale, i.e., the support scale for scalar gradients [16]. In this Rapid Communication we introduce the concept of the mixing scale based on the distribution of pair separations. We study the evolution of the mixing scale due to the competition of shear action and diffusion and relate it to the anomalous evolution of the global dispersion scale.

(a) Mixing, coarse-grained, and dispersion scales. Mixing can be studied in terms of pair dispersion, which quantifies the evolution of the mean square separation between two particles with a given initial separation [15]. For turbulent flows, this can be directly linked to the decay of scalar variance [17]. However, it does not provide in itself a measure of a local mixing scale, defined as the support scale for concentration gradients. A characteristic scale that can be defined for random mixtures is the coarse-grained scale $\eta_{q}$ [16], which is the scale $r$ at which the variance of the coarse-grained concentration

$$
c_{r}(\mathbf{x}, t)=\frac{1}{r^{d}} \int_{\rho<r} d^{d} \rho c(\mathbf{x}+\boldsymbol{\rho}, t)
$$

decreases by a factor $q$ from its point value. The coarse-grained scale $\eta_{q}$ reflects the aggregate construction of the concentration field. By definition $\eta_{q}$ does not conserve the full concentration variability and it can be much larger than the support scale of concentration gradients [16].

In order to define a local mixing scale that conserves the full variability of a scalar field, we consider the probability density function (PDF) of particle separations,

$$
p(\rho, t)=\left\langle\delta\left\{\rho-\left[\mathbf{x}^{(1)}(t)-\mathbf{x}^{(2)}(t)\right]\right\}\right\rangle,
$$

where $\mathbf{x}^{(i)}(t)(i=1,2)$ denotes the position of two particles at time $t$ and the angular brackets denote the average over all particle pairs. The cumulative distribution of absolute separations $r$ is

$$
P(r, t)=\int_{|\rho|<r} d \rho p(\rho, t) .
$$

For a uniform distribution of particles, $P(r, t)$ scales as $r^{d}$ because the number of particles within a hypersphere of dimension $d$ and radius $r$ is proportional to the volume of 
the hypersphere. Therefore, at the well mixed scales, the normalized cumulative distribution

$$
C(r, t)=P(r, t) r^{-d}
$$

is constant. For a nonuniform distribution of particles, the evolution of $P(r, t)$ is typically slower than $r^{d}[18]$ such that $C(r, t)$ decreases with $r$. We define the mixing scale $\epsilon$ as the transition scale for which the normalized cumulative distribution shifts from uniform $[C(r, t)=$ const.] to nonuniform,

$$
\epsilon(t)=\max [r \mid C(r, t)=\text { const. }] .
$$

Unlike classical pair-dispersion studies, the mixing scale here is defined from a transition scale analysis rather than a spatial moment analysis.

It can be shown that $C(r, t)$ is equal to the variance of the coarse-grained concentration [Eq. (1)] by noting that the cumulative distribution of pair separation is equal to the correlation integral defined by Grassberger and Procaccia [18]. For scales $r<\epsilon, C(r, t)$ is independent of $r$ and equal to the point value concentration variance. Therefore, $\epsilon$ is the scale that preserves the full concentration variance. The state of incomplete mixing of a scalar field can be characterized by comparing the mixing scale $\epsilon(t)$ to the characteristic dispersion scale $\sigma(t)$, which quantifies the size of the plume. $\sigma(t)$ is defined by the second spatial moment of pair separation

$$
\sigma^{2}(t)=\int d \rho \rho^{2} p(\boldsymbol{\rho}, t) .
$$

(b) Spatially uniform shear and local balance rule. For spatially uniform shear, the temporal evolution of the mixing scale $\epsilon$ is governed by the competition of expansion due to diffusion and compression due to shear [16]. This can be expressed by a local balance rule

$$
\frac{1}{\epsilon} \frac{d \epsilon}{d t}=\frac{D}{\epsilon^{2}}-\gamma,
$$

where $D$ is the diffusion coefficient and $\gamma$ is the compression rate.

(c) Heterogeneous flow and nonlocality. For a heterogeneous flow field, the shear varies in space. Therefore, the size of well mixed areas is spatially distributed (Fig. 1). The latter is typically minimum in zones of maximum shear. By Eq. (5), the mixing scale $\epsilon$ corresponds to the minimum size of the well mixed patches in the domain. As the concentration distribution is transported through the medium, the location of the well mixed zone with minimum size changes. Thus the time evolution of $\epsilon$ no longer depends on a local balance of diffusion and shear and it becomes nonlocal.

In the following we analyze the temporal evolution of the mixing scale for transport in steady flows through heterogeneous media (Fig. 1). While the mixing characteristics are generally different from temporally fluctuating random flows, the basic mechanisms such as creation and dissipation of scalar gradients are similar. Unlike turbulent velocity fields, flow patterns are not transported by the mean flow and the Taylor hypothesis is not valid. For strong degrees of heterogeneity, dispersion, as measured by $\sigma(t)$ [Eq. (6)], is superdiffusive as a result of the non-Gaussian, heavy tailed distribution of Lagrangian velocities and their long range temporal correlation [1,19-21]. The temporal behavior of the mixing scale, on the other hand, is not known in this context. Mixing is generally expected to be enhanced in strongly heterogeneous flow fields $[7,22,23]$. Thus the mixing scale may be expected to grow superdiffusively too. In the following we show that its temporal growth is, on the contrary, slowed down by the flow disorder.

To analyze the temporal evolution of the mixing scale, we perform high resolution numerical simulations of transport through heterogeneous porous media using a standard approach [21]. The medium conductivity $K(\mathbf{x})$ is modeled as a lognormally distributed random field, i.e., the joint distribution of the point values of logarithmic conductivity $\ln K$ is multivariate Gaussian and characterized by a correlation length $\lambda$ and a variance $\sigma_{\ln K}^{2}$ [Fig. 1(a)]. The correlation function $C(\mathbf{x})$ of $\ln K$ fluctuations is $C(\mathbf{x})=\exp \left(-\mathbf{x}^{2} / 2 \lambda^{2}\right)$. This type of random conductivity is generic and represents a reference field for theories of flow and transport in heterogeneous porous media [24].

Spatial variability of the conductivity $K(\mathbf{x})$ is mapped onto the divergence-free flow field $\mathbf{u}(\mathbf{x})$ via the Darcy equation $\mathbf{u}(\mathbf{x})=-K(\mathbf{x}) \nabla h(\mathbf{x})$, with $h(\mathbf{x})$ the hydraulic head. As boundary conditions we impose no flux across the horizontal and a constant hydraulic head at the vertical boundaries. The flow equation is solved numerically with a finite difference scheme. The flow domain is the two-dimensional rectangle $\Omega=\left\{\mathbf{x} \mid x_{1} \in[0,819.2 \lambda], x_{2} \in[0,102.4 \lambda]\right\}$. The strong heterogeneity of the conductivity field and the flow equations (Darcy equation and fluid mass conservation) imply a strong localization of high velocity zones such that the flow field organization is different from the conductivity field organization [21]. The flow correlation range is long range in the longitudinal direction and short range in the transverse direction [Fig. 1(b)].
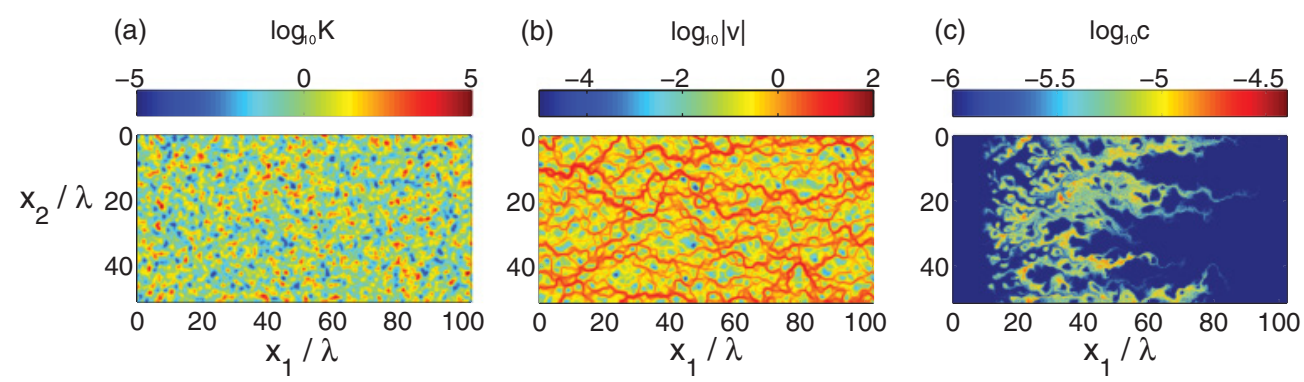

FIG. 1. (Color online) (a) Lognormal conductivity field with variance $\sigma_{\ln K}^{2}=9$ and correlation length $\lambda=10$. (b) Simulated velocity field. (c) Simulated scalar field $C(x, t)$ at time $t=20 \tau_{a}$ for an initial line injection at $x_{1} / \lambda=10$ and a Péclet number Pe $=10^{2}$. 
Transport of a passive scalar $c(\mathbf{x}, t)$ in the flow field $\mathbf{u}(\mathbf{x})$ can be described by the Fokker-Planck equation

$$
\frac{\partial c(\mathbf{x}, t)}{\partial t}+\mathbf{u}(\mathbf{x}) \cdot \nabla c(\mathbf{x}, t)-D \nabla^{2} c(\mathbf{x}, t)=0,
$$

where $D$ denotes the (constant) diffusion coefficient. As boundary conditions we impose a flux weighted instantaneous line injection at the inlet at $x_{1}=0$, no flux at the horizontal boundaries, and an absorbing boundary at the outlet. The characteristic advection time scale is defined by $\tau_{a}=\lambda / \bar{u}$, where $\bar{u}$ is the mean transport velocity. The characteristic diffusion time is $\tau_{D}=\lambda^{2} / D$. The Péclet number Pe $=\lambda \bar{u} / D$ compares the diffusive and advective time scales. Equation (8) is solved by random walk particle tracking [20,21]. Figure 1(c) displays an example of a simulated scalar distribution for a large conductivity field variance $\sigma_{\ln K}^{2}=9$ and a Péclet number of $\mathrm{Pe}=10^{2}$, which implies a velocity field that varies over seven orders of magnitude [20,21]. On the forward edge, the scalar field is characterized by a filamentary structure due to the meandering high velocity channels. The backward edge is composed of scalar blobs that remain trapped in low velocity zones. These low velocity areas create low concentration lacunarities within the scalar field. Both the forward fingering and backward trapping imply that the dispersion scale evolves superdiffusively.

The spatial structure of the scalar field is quantified by the normalized cumulative PDF of particle separations $C(r, t)$ [Eq. (4)], shown in Fig. 2 for different travel times. At a given time, $C(r, t)$ displays three spatial scaling regimes. In the first regime, $C(r, t)$ is independent of $r$. This is the homogeneous range, where concentration gradients are erased by diffusion. In the second regime, $C(r, t)$ decreases with scale $r$. Its slope on a log-log plot reflects the dimensionality of the concentration field, as may be quantified by the second order fractal dimension [18]. This slope decreases with time, which reflects the smoothing of the concentration

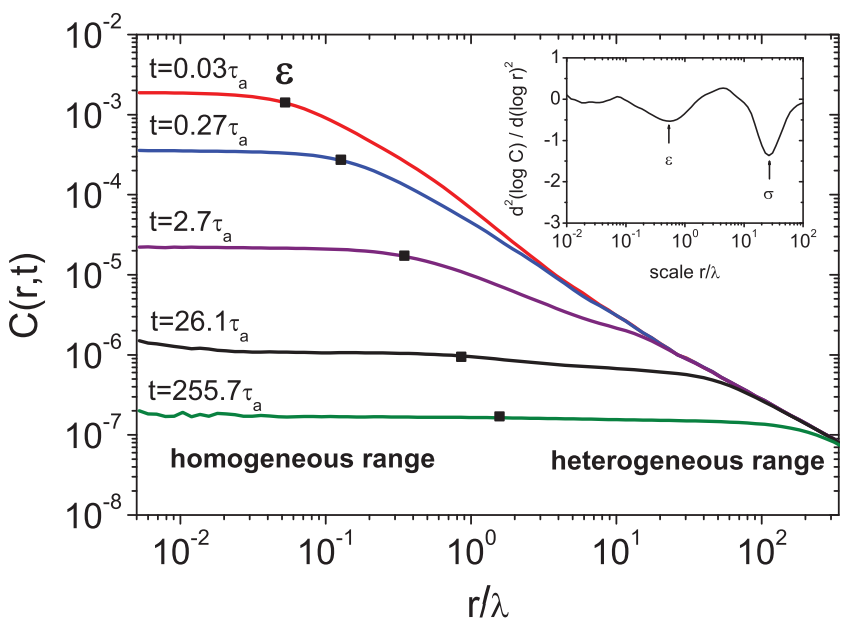

FIG. 2. (Color online) Normalized correlation integral $C(r, t)$ as a function of the radial scale $r$ at times $t=0.03 \tau_{a}, 0.27 \tau_{a}, 2.7 \tau_{a}$, $26.1 \tau_{a}$, and $255.7 \tau_{a}$; the small scale value of $C(r, t)$ decreases with time. The inset shows the second derivative of the normalized correlation integral $\partial^{2} \log _{10} C(r, t) / \partial \log _{10} r^{2}$ as a function of the scale $r$ for time $t_{7}=8.4 \tau_{a}$. The two minima are found for $r=\epsilon$ and $\sigma$, which correspond to the changes of scaling regimes.
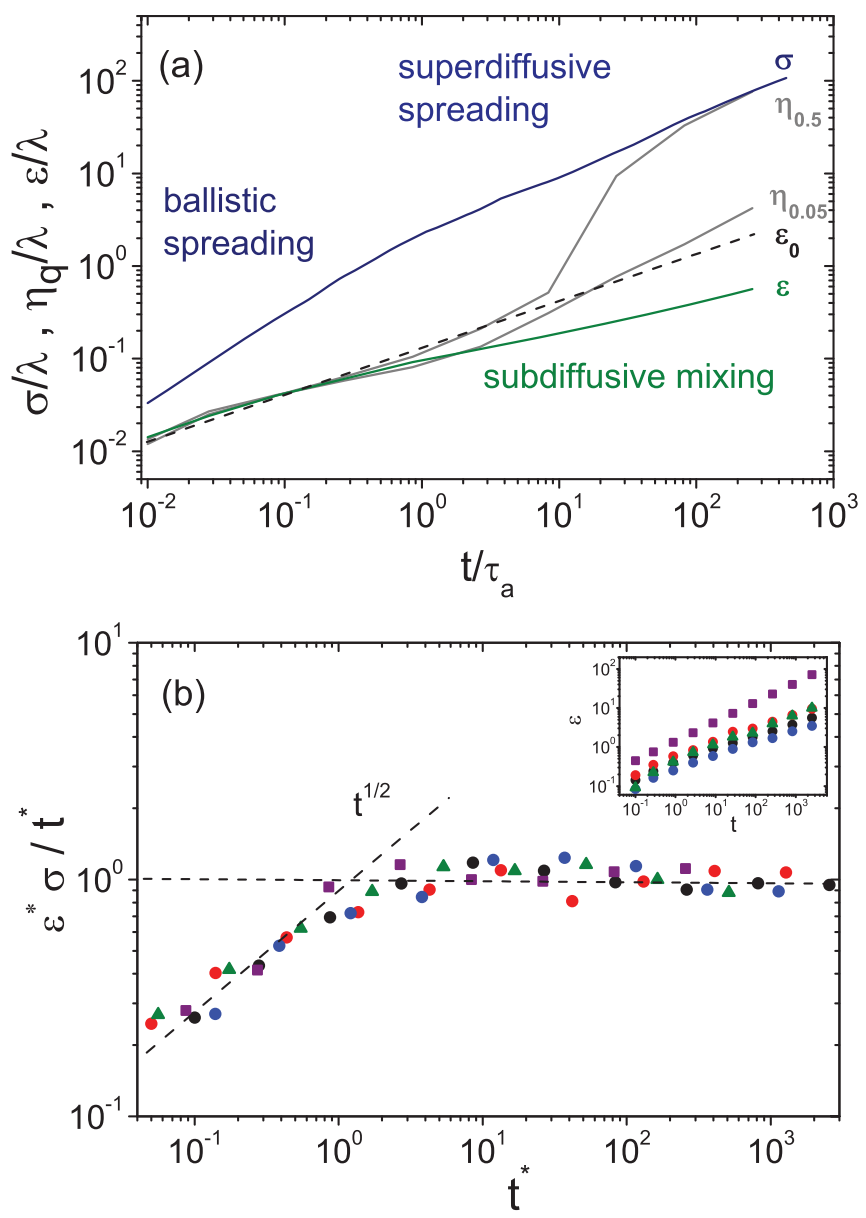

FIG. 3. (Color online) (a) Temporal evolution of the dispersion scale $\sigma$, the mixing scale $\epsilon$, and coarse-grained scales $\eta_{0.05}$ and $\eta_{0.5}$. The diffusion scale $\epsilon_{0}=\sqrt{2 D t}$ is represented by the dashed line. The scales $\epsilon$ and $\eta_{q}$ are normalized to be equal to the diffusion scale at the smallest time $t=10^{-2} \tau_{a}$. (b) Synthesis of simulation data obtained for correlation lengths ranging from $\lambda=5$ to 20 , Péclet numbers ranging from $P e=10$ to 100 , and logarithmic permeability variances ranging from $\sigma_{\ln K}^{2}=1$ to 9 . The time $t^{*}$ and the mixing scale $\epsilon^{*}$ are normalized so that the transition between the two regimes is unity. The inset shows the temporal evolution of $\epsilon$ for the different parameter sets.

field as time increases. We call this range the heterogeneous range (see Fig. 2). As defined above, the mixing scale $\epsilon$ [Eq. (5)] separates the homogeneous and heterogeneous ranges. It is estimated here from the minimum of the second derivative $\partial^{2} \log _{10} C(r, t) / \partial \log _{10} r^{2}$. The inset in Fig. 2 shows that this criterion allows for a sharp identification of the transition between the homogeneous and heterogeneous regimes.

The temporal evolution of the mixing scale $\epsilon(t)$ is compared to that of the global dispersion scale $\sigma(t)$ in Fig. 3(a). At initial times, the dispersion scale evolves ballistically, reflecting the growth of the plume extent due to purely advective mechanisms, $\sigma^{2}(t)=\sigma_{u}^{2} t^{2}$, where $\sigma_{u}^{2}$ is the velocity variance. The mixing scale evolves diffusively, $\epsilon^{2}(t)=2 D t$, since the plume is too small to sample the velocity contrasts. For times larger than the advection scale $\tau_{a}$, dispersion and mixing become coupled since scalar particles start sampling 
the velocity heterogeneity. As a consequence, the dispersion scale evolves superdiffusively and the mixing scale, in contrast, grows subdiffusively, a behavior not described by the local balance rule [Eq. (7)].

In the subdiffusive mixing regime, the difference between $\sigma$ and $\epsilon$ reflects the fact that the concentration field is not fully mixed within the plume. The difference between these two scales diverges with time by as much as two orders of magnitude at late times, as illustrated in Fig. 3(a). The two scales $\epsilon$ and $\sigma$ are found to be related as

$$
\epsilon \sigma \propto t \text {. }
$$

This relationship expresses the coupling between mixing and dispersion via the velocity field organization. Beyond the illustrative example shown in this Rapid Communication we tested this result for a variety of parameters that are compatible with our numerical setting [Fig. 3(b)] and found that this relationship held in all test cases: $\epsilon \sigma / t=$ const. for $t>\tau_{a}$ (the coupled regime) and $\epsilon \sigma / t \propto t^{1 / 2}$ for $t<\tau_{a}$ (the uncoupled regime).

The temporal evolution of the coarse-grained scales $\eta_{q}(t)$, with $q=0.05$ and 0.5 , is displayed in Fig. 3 for comparison with the mixing scale $\epsilon(t)$. At late times the scale $\eta_{q}$ tends toward the global dispersion scale at a rate that depends on $q$. This supports the notion that mixing is enhanced by flow heterogeneity. However, when defining the mixing scale $\epsilon$ from a detailed transition scale analysis, one finds that the support scale for concentration gradients is much smaller than the coarse-grained scales (up to two orders of magnitude). This implies that, although the concentration field appears smooth and diffuse within the mixing zone, the maximum fully mixed support volume remains small.

The persistent incomplete mixing demonstrated here gives constraints on assumptions that can be made when modeling reactive processes in disordered media. For broad velocity gradient distributions, local balance approaches based on Eq. (7) are not applicable to the description of the evolution of the mixing scale, as the competition between diffusion and shear is nonlocal. The mixing scale $\epsilon(t)$ evolves subdiffusively for a large range of times and diverges from the typical dispersion size that grows superdiffusively. The scaling complementarity between dispersion and mixing [Eq. (9)] provides a simple relationship between anomalous global dispersion and local mixing dynamics.
[1] J. P. Bouchaud and A. Georges, Phys. Rep. 195, 127 (1990).

[2] R. Kubo, M. Toda, and N. Hashitsume, Statistical Physics II, Non-Equilibrium Statistical Mechanics (Springer-Verlag, Berlin, 1991).

[3] M. Dentz and D. Bolster, Phys. Rev. Lett. 105, 244301 (2010).

[4] A. M. Tartakovsky, D. M. Tartakovsky, and P. Meakin, Phys. Rev. Lett. 101, 044502 (2008).

[5] K. Kang and S. Redner, Phys. Rev. Lett. 52, 955 (1984).

[6] A. J. McKane and T. J. Newman, Phys. Rev. E 70, 041902 (2004).

[7] A. Sokolov, R. Goldstein, F. Feldchtein, and I. Aranson, Phys. Rev. E 80, 031903 (2009).

[8] S. B. Pope, Tubulent Flows (Cambridge University Press, Cambridge, 2000).

[9] J. Ottino, Annu. Rev. Fluid Mech. 22, 207 (1990).

[10] A. Venaille and J. Sommeria, Phys. Rev. Lett. 100, 234506 (2008).

[11] Z. Neufeld, C. López, and P. H. Haynes, Phys. Rev. Lett. 82, 2606 (1999).

[12] T. Benzekri, C. Chandre, X. Leoncini, R. Lima, and M. Vittot, Phys. Rev. Lett. 96, 124503 (2006).
[13] N. Kleinfelter, M. Moroni, and J. H. Cushman, Phys. Rev. E 72, 056306 (2005).

[14] P. K. Kitanidis, Water Resour. Res. 30, 7 (1994).

[15] M. Bourgoin, N. Ouellette, H. Xu, J. Berg, and E. Bodenschatz, Science 311, 835 (2006).

[16] E. Villermaux and J. Duplat, Phys. Rev. Lett. 97, 144506 (2006).

[17] M. Nelkin and R. M. Kerr, Phys. Fluids 24, 1754 (1981).

[18] P. Grassberger and I. Procaccia, Phys. Rev. Lett. 50, 346 (1983).

[19] B. Berkowitz, A. Cortis, M. Dentz, and H. Scher, Rev. Geophys. 44, RG2003 (2006).

[20] T. Le Borgne, M. Dentz, and J. Carrera, Phys. Rev. E 78, 026308 (2008).

[21] T. Le Borgne, M. Dentz, and J. Carrera, Phys. Rev. Lett. 101, 090601 (2008).

[22] A. V. Straube and A. Pikovsky, Phys. Rev. Lett. 99, 184503 (2007).

[23] A. Libal, C. Reichhardt, and C. J. Olson Reichhardt, Phys. Rev. E 78, 031401 (2008).

[24] X. Sanchez-Vila, A. Guadagnini, and J. Carrera, Rev. Geophys. 44, 1029/RG3002 (2006). 